\title{
A Study on Laser Micromachining
}

\author{
Muhammad Sajjad ${ }^{1}$, Jithin Ambarayil Joy ${ }^{1}$ and Dong Won Jung1,a \\ ${ }^{1}$ Department of Mechanical Engineering, Jeju National University, Jeju, South Korea
}

\begin{abstract}
Ultra Violet (UV) pulse are widely used for micromachining in the research and development of Information Technology (IT), Nano Technology (NT), and Bio Technology (BT) products because due to short wavelength of the laser it not only allows micro drilling, micro cutting and micro grooving with high accuracy, but acts also as high penetrating coefficient, which give opportunity for different materials to machined properly. A finite element model considering photo-thermal and photomechanical properties was recommended to study the micro phenomena subject by mechanical pressure impacts and thermal heat transfers during a very short processing time. From the results of this research work, few of dynamic deformation behaviours such as deformation shape, strain, and stress distributions were examine and compare it with the experimental results of previous research work. The results from this comparison will help to know better knowledge about interference of UV laser beam and metal materials.
\end{abstract}

Keywords: UV pulse, Photothermal, Photomechanical, Deformation shape, Stress distributions.

\section{INTRODUCTION}

It is established that nanosecond pulsed laser processing of metals, ceramics, and polymer materials is a complex phenomenon associated with photochemical, photothermal and photomechanical effects. Photothermal effects are the dominant phenomena in processing metals and photochemical effects are dominant in processing polymers, especially under high-density lasers. During laser irradiation on the surface of the material, some parts of laser light are reflected to the air or transmitted and the remainder is absorbed inside the material. Absorbed energy is transferred to the inside of the material by thermal conduction, and then the material melts in a very short time. When the amount of energy increases, some of material is rapidly removed by explosive vaporization with chemical and physical decomposition, this process is called the photothermal ablation mechanism [1].

Recently the study of LSP(Laser Shock Processing) on micro copper and aluminum were presented by Wenwu Zhang and others. A comparative study on experimental and computational analysis about micromachining using micro beam laser was reported using LSP. As soon as the strong-pulsed laser beam arrives and focuses on the surface of materials, the surface layer evaporates immediately and the plasma is generated. This plasma induces shock waves from the irradiated surface during the air expansion, and the mechanical impact pressures are transferred to the target. If it is confined by water, or another type of medium, the shock pressure of being under-water can be over five times that of open-air. The medium, water, also protects the target materials from thermal energy so that only pure mechanical energy effects the processing [2,3]. When lasers irradiate the surface of metals, the impact processing caused by shockwave pressure is called the photomechanical ablation mechanism. When laser micromachining is processed in nanoseconds, the average strain rate was approximately 104 105(sec-1), which is very high [5].
Therefore, analysis results which do not considered the strain rate effect show a lot of difference with the experimental results. So far, the theoretical analyses for machining metals using UV lasers with wavelengths between 200 and 400nm have studied the photothermal effect to predict ablation depth and diameter. However, the behavior of molten metal and the analysis of temperature distribution are very complex problems because there are a lot of process parameters such as convection, radiation and conduction and it occurs for a very short time. So, the behavior is difficult to predict accurately because of the wide variance between model and experiment.

In this paper, a computational analysis of photothermal ablation of copper using UV pulse laser was performed in two parts for thermal and mechanical effects. Then, a new finite element model that combined photothermal and photomechanical effects was suggested. Finally, the analyzed results of this new model were compared and verified with previous experiment results.

\section{FINITE ELEMENT MODEL}

A $355 \mathrm{~nm}$ UV pulse laser was used to perform this experiment and model analysis the layout of this system is shown in Fig.1. Copper material was used in this analysis, the model has a $40 \mu \mathrm{m}$ diameter and thickness of $20 \mu \mathrm{m}$. Each analysis termination time in this analysis was 50 nanoseconds, and the pulse duration time was 25 nanoseconds. The model was composed of total 16,544 elements and mesh relevance was chosen at the value of +100 in order to improve the accuracy of analysis results. Only $1 / 4$ of the copper thin film was modeled due to its symmetric geometry. The metric units used $\mu \mathrm{m}-\mathrm{kg}-\mu \mathrm{N}-\mathrm{s}-$ system and the mm-tone-N-s-K units for ANSYS and LSDYNA, respectively. Table 1 shows the mechanical and Thermo-physical properties of copper material [6].

\footnotetext{
${ }^{a}$ Professor Dong-Won Jung, Department of Mechanical Engineering, Jeju National University, Jeju, Republic of Korea : jdwcheju@jejunu.ac.kr
} 


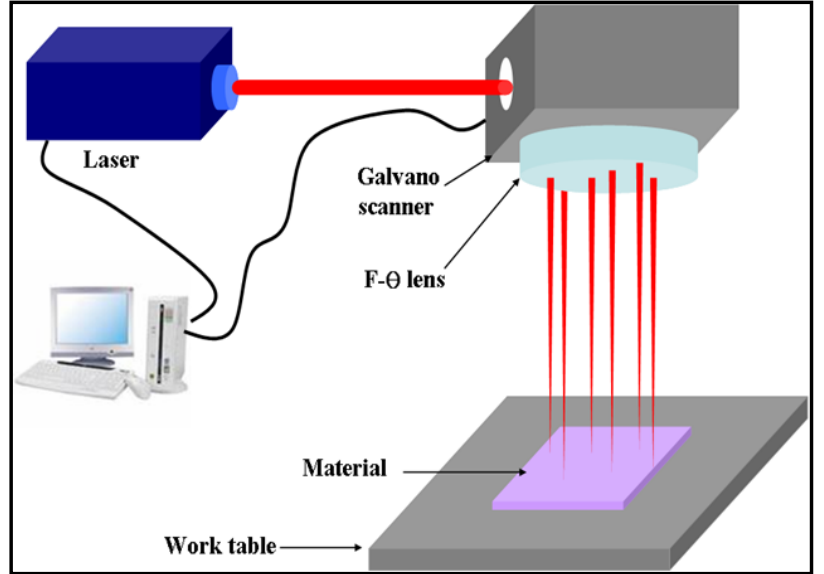

Figure 1. Schematics of Galvano scanner and work table.

At first, transient thermal analysis and static structure analysis of thermal stress was performed in one coupled environment of ANSYS Workbench, and then explicit dynamic analysis was introduced from the result of ANSYS, and finally executed as finite element analysis tool using LS-DYNA program. The FEM analysis procedure is shown in Fig. 2

Table 1. Mechanical and Thermo-Physical Properties of Copper Material.

\begin{tabular}{|l|l|}
\hline Density $(\mathrm{kg} / \mathrm{m} 3)$ & 8,300 \\
\hline Elastic Modulus $(\mathrm{Pa})$ & $1.1 \times 10^{11}$ \\
\hline${ }^{\mathrm{U}}$ (Poisson's ratio) & 0.34 \\
\hline Yield Strength $(\mathrm{Pa})$ & $2.8 \times 10^{8}$ \\
\hline $\begin{array}{l}\text { Isotropic Thermal Conductivity }(\mathrm{W} / \mathrm{m} / \\
\left.{ }^{\circ} \mathbf{C}\right)\end{array}$ & 401 \\
\hline Specific Heat $\left(\mathrm{J} / \mathrm{kg} /{ }^{\circ} \mathbf{C}\right)$ & 385 \\
\hline Coefficient of Thermal Expansion $\left({ }^{\circ} \mathbf{C}^{-1}\right)$ & $1.85 \times 10^{-5}$ \\
\hline Reference Temperature $\left({ }^{\circ} \mathbf{C}\right)$ & 22 \\
\hline
\end{tabular}

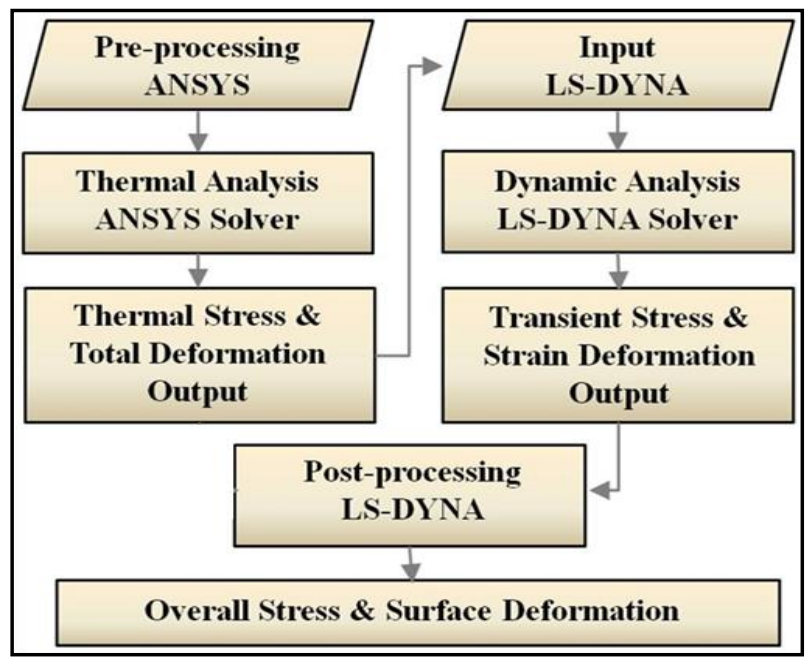

Figure 2. FEM analysis procedures
The photomechanical analysis model was considered under the following assumptions:

- The impact pressure wave is uniaxial and planar.

- The pressure produced due to the laser pulse is uniform.

- The material obeys the Von-Misses yielding criterion.

- The viscous effect is ignored.

The Cowper-Symonds relation was used in this analysis model when considering the strain rate effect in pulse laser processing. The relation equation was the constitutive equation of the copper material, represented by multiplying the strain hardening by the strain rate hardening. The peak pressure, 3,350 $\mathrm{MPa}$, generated by the expansion of plasma was referenced by previous research.

\subsection{Photo-thermal Analysis}

From the results of transient thermal analyses, the distribution of temperature, heat flux, and other thermal quantities were determined over the total analysis time. In order to obtain more accurate transient analysis simulating real phenomena, it was very important to input the quantity of heat flux by introducing the graph of the relationship between heat flux and time for each time period. Initial temperature conditions could be adapted as uniform or non-uniform thermal condition, but the temperature of the copper surface was assumed as room temperature of $22^{\circ} \mathrm{C}$ in this analysis model because only single pulsed ablation was considered in this analysis.

The absorption of copper is assumed at approximately 70 percent when the laser wavelength $(\lambda)$ was $355 \mathrm{~nm}$. Heat flux was applied and represented as distributed thermal load and transfer heat flow per area and time. The positive value means that energy will come into the model, while the negative means that energy will come out of the model. The values of thermal conductivity, density, and specific heat of copper material had to be defined for a transient thermal analysis. Thermal conductivity was assumed as isotropic in this model. The thermo-physical properties of copper are shown in Table 2 .

\section{EXPERIMENT AND ANALYSIS RESULTS}

\subsection{Photo-thermal and Photomechanical Analysis Results}

Thermal stress distributions and dilated deformation were obtained through photo-thermal analysis as shown in Fig. 3. The surrounding of the dent was raised up about 0.09 $\mu \mathrm{m}$ because of thermal expansion. The scale was magnified to easily identify the raised part as shown in Figure 3 . The impact pressure analysis was accomplished after introducing the results of thermal analysis, including the raised surface geometry data, and the analysis procedure was coupled with ANSYS and LS-DYNA programs. With laser shock waves analysis, the pressure wave generated during the pulse time of 25 nanoseconds strongly impacted the surface and then a dent was formed with a very small area.

\subsection{Photomechanical Analysis}




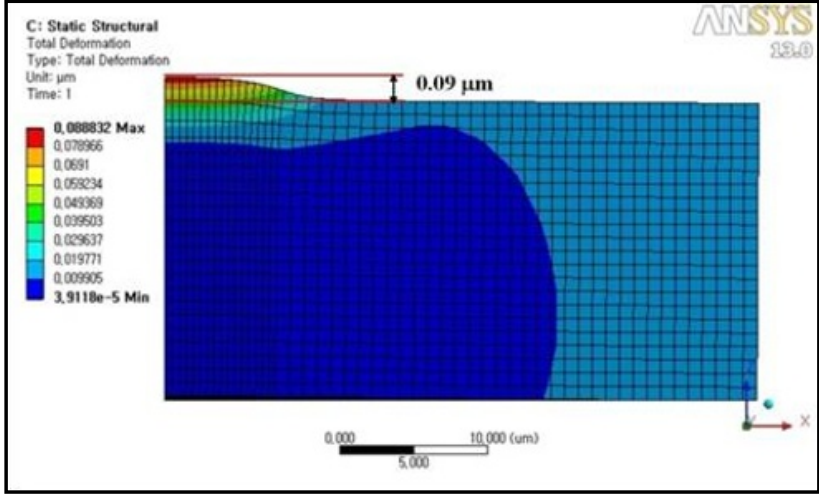

Figure 3. Total deformation of dilatational surface.

\subsection{Photomechanical Analysis Results without the strain rate effect}

The effective von-Mises stress and plastic strain distribution, ignoring the strain rate effect, are shown in Figs.4. After 50 nanoseconds, pressure of $500 \mathrm{MPa}$ was calculated in the model. It was also found that the dent diameter and depth was about $16 \mu \mathrm{m}$ and $7.24 \mu \mathrm{m}$, respectively. The burr height around the dent was measured at about $1.5 \mu \mathrm{m}$. The analysis model without strain rate effect was more locally deformed than the experimental results on the surface of the laser beam focused area.

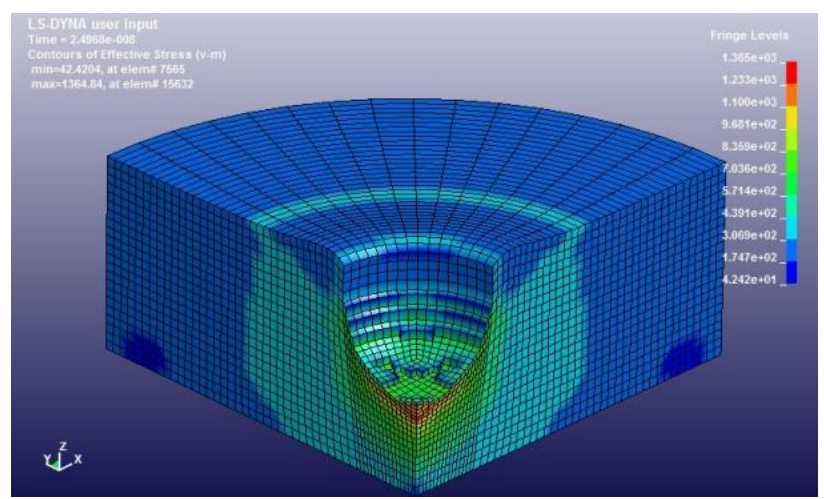

Figure 4 Effective stress ( $v-m)$ distributions for copper without the strain rate effect

\subsection{Photomechanical Analysis Results with the strain rate effect}

Figure 5 show the effective von-Mises stress and plastic strain distribution with the strain rate effect. After 50 nanoseconds, 3,500 $\mathrm{MPa}$ of pressure was calculated in the model. In this case the diameter and depth of the dent was about $11.9 \mu \mathrm{m}$ and $0.64 \mu \mathrm{m}$, respectively. If the strain rate increases, the fracture time and the strength of material increase because of the strain-rate hardening effect. The maximum stress in the model considering the strain rate effect was higher than non-considering model, and the burr around the dent was barely observed, despite strong laser pressure. Deformation energy from the laser shock wave was absorbed over the whole material. The SEM picture of the real dent processed by LSP was also compared with two FE analysis models, with and without strain-rate effect. When compared and measured between the analysis and experimental results, the proposed model considering strain rate effect was more effective in predicting micro dent shape processed by laser shock wave.

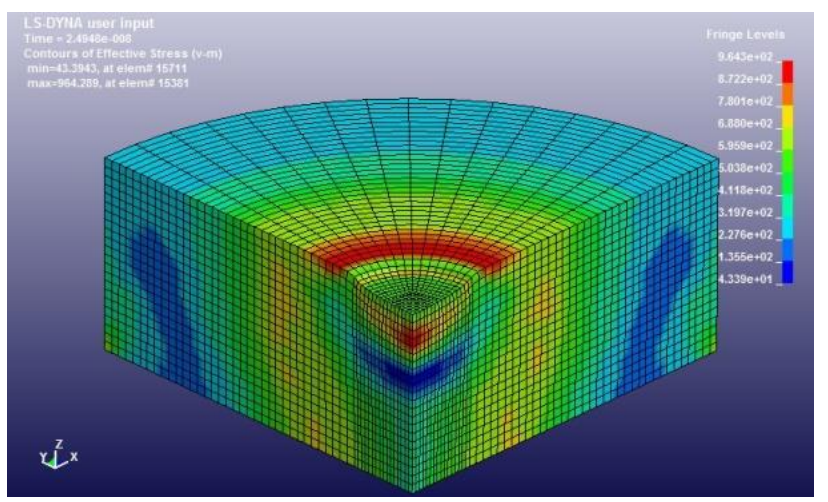

Figure 5. Effective stress (v-m) distributions for copper with the high strain rate effect

\subsection{Experiment Results}

SEM pictures of experimental results of thin copper sheets with respect to the change in fluence of the $355 \mathrm{~nm}$ UV laser are shown in Figure 6 . When processed at a fluence of $0.23 \mathrm{~J} / \mathrm{cm}^{2}$, the middle part irradiated and melted by the laser beam was raised up a little as shown in Fig. 6(a). The small crater in the middle of the raised part, as shown in Fig. 6 (b), was created when processed with the fluence of $0.8 \mathrm{~J} / \mathrm{cm}^{2}$. That is, the threshold energy capable of removing the metal can be determined as a fluence of $0.8 \mathrm{~J} / \mathrm{cm}^{2}$. Small crown-like craters formed by copper melted and pushed out were shown with the fluence of $13.56 \mathrm{~J} / \mathrm{cm}^{2}$ as shown in Fig. 6 (c). Generally, photo-thermal ablation provided the thermal expansion of material at low laser fluence, which was almost near to threshold energy. On the other hand, the burr consisted of particles oxidized by both re-solidification of the melt and re-condensation of evaporated material, which were remained near the crater hole at the high fluence. In this paper, the coupled analysis model was introduced with a fluence of $0.8 \mathrm{~J} / \mathrm{cm}^{2}$ to compare with simulated and experimental dent shapes.

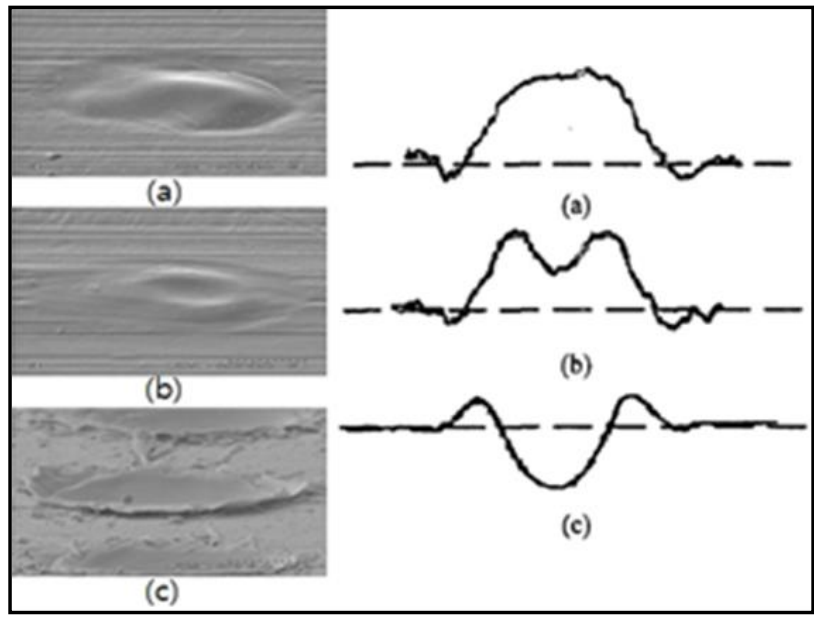

Figure 6. SEM photos of copper processed by UV laser under the fluence of (a) $0.23 \mathrm{~J} / \mathrm{cm} 2$, (b) $0.8 \mathrm{~J} / \mathrm{cm} 2$, and (c) 13.56 $\mathrm{J} / \mathrm{cm} 2$ with 30 shots, respectively 


\section{CONCLUSION}

From these experiments and analyses, we reached the following conclusions. The photomechanical analysis model that considers the effect of high strain-rate was very useful in predicting the LSP ablation phenomena and confirmed by comparison with experimental results. Photo-thermal ablation problem was performed using transient thermal analysis so that the maximum temperature was observed on the surface of copper sheet, which was a little above of the melting point of work piece material. Threshold energy condition of laser ablation was therefore found, which meant the starting point of material removal for laser micromachining process. Finally a new simulation model for photomechanical analysis coupled with photo-thermal effects was suggested and the results were verified with excellent agreement with the real ablation.

\section{References}

1. A. Mecke, I. Lee, J.R. Baker jr., M.M. Banaszak Holl, B.G. Orr, Eur. Phys. J. E 14, 7 (2004)

2. A M. Ben Rabha, M.F. Boujmil, M. Saadoun, B. Bessaïs, Eur. Phys. J. Appl. Phys. (to be published

3. A J. Y. Oh, B. S. Shin, J. of KSPE 24, 4 (2007)

4. A W. Zhang, Y. L. Yao, J. of Manuf. Process 3, 2 (2001),

5. A T. Hu, Z. Yao, J. Hu, Surf. \& Coat. Technol. 201, (2006)

6. A B. S. Shin, T. J. J, J. of KSPE 5, 2 (2006 